\section{CRITICAL SUPRAVALVULAR AORTIC STENOSIS IN A FAMILY WITH A NEW ELASTIN GENE MUTATION}

A. Jakob ${ }^{1}$, S. Unger ${ }^{2}$, R. Arnold ${ }^{1}$, J. Grohmann ${ }^{1}$, B. Stiller ${ }^{1}$

${ }^{1}$ Pediatric Cardiology, ${ }^{2}$ Human Genetics, University Hospital of Freiburg, Freiburg im Breisgau, Germany

Background and aims: An elastin gene mutation causes manifold vascular stenosis in patients with Williams-Beuren Syndrome, but also in a nonsyndromatic congenital form. These patients have a higher risk of sudden death, particularly when undergoing diagnostic or surgical procedures.

Methods: We performed Molecular genetic investigation of the elastin - Gen in one family. The cardiac and vascular malformations in these patients were determined and 2 lethal clinical courses are described.

Results: Screening over 3 generations revealed 8 affected individuals with a new Elastin - gen mutation. The cardiac and vascular malformations range from mild asymptomatic supravalvular aortic stenosis and isolated dysplastic atrioventricular valves to diffuse arterial hypoplasia. Two infants presented arteries affected at multiple locations, including the left coronary artery. Both died of sudden heart death and myocardial ischemia, one while under general anesthesia for cardiac catheterization, the other perioperatively.

Conclusions: In patients with arterial narrowing, elastin - Gen mutation should be considered. These patients have special pathophysiological aspects. Any application of general anesthesia should undergo careful scrutiny and only be performed by well-experienced anesthesiologists anticipating such problems.

\section{CARDIAC EVALUATION OF NEONATES WITH IMPAIRED INTRAUTERINE GROWTH}

E.H. Aburawi ${ }^{1}$, A. Thuring ${ }^{2}$, P. Malcus ${ }^{2}$, A. Maxedius ${ }^{3}$, E. Pesonen ${ }^{3}$

${ }^{1}$ Paediatric, Faculty of Medicine and Health Science, United Arab Emirates University, Al-Ain, United Arab Emirates, ${ }^{2}$ Obs. and Gynecology, ${ }^{3}$ Paediatric Cardiology, Lund University, Lund, Sweden

Background and aims. We investigated the relationship between the cardiac function and coronary flow in neonates with impaired intrauterine growth.

Methods. Fetal growth was assessed by fetometry and Doppler velocimetry of the umbilical artery blood flow. Impaired fetal growth was defined as an estimated fetal weight less than mean -2SD from the normal gestational age related fetal weight. Cardiac function and left anterior descending artery (LAD) flow parameters were measured by transthoracic Doppler echocardiography in 14 newborns at one week of age when the ductus arteriosus was closed. Gestational age- and age-matched healthy newborns, appropriate for gestational age $(\mathrm{N}=15)$ served as controls.

Results. The mean pulsatility index was 0.97 and the mean birth weight 2.2 (range 2.1 to 2.4 ) kg. The left ventricular shortening fraction was normal $39 \pm 4.3 \%$. Aortic velocity time integral/minute as a measure of systolic LV function correlated to LAD $P F V d, r=0.54, p<0.0001$. LAD peak flow velocity in diastole (PFVd) correlated to left ventricular mass $(r=0.46, p=0.0001)$. The mean LAD diameter was $0.99 \pm 0.09 \mathrm{~mm}$. Impaired intrauterine growth was associated with an increase of PFVd (mean $34.5 \pm 4$, controls $19 \pm 6 \mathrm{~mm} / \mathrm{s}, \mathrm{p}=0.0001$ ) and coronary flow $(7.3 \pm 2$, and $4.8 \pm 2 \mathrm{ml} / \mathrm{min}$ respectively, $p=0.04)$. $\mathrm{LAD}$ velocity time integral per minute correlated to mitral peak E-wave $(r=0.74, p<0.01)$.

Conclusions. Basal coronary flow and peak flow velocity appear to be significantly increased in these neonates with intrauterine growth impairment, which leads to decreased coronary flow reserve. LAD flow parameters were linearly related to LV systolic and diastolic functions and to LV mass. 